\title{
Supplementation with Branched-Chain Amino Acids Induces Unexpected Deleterious Effects on Astrocyte Survival and Intracellular Metabolism with or without Hyperammonemia: A Preliminary In Vitro Study
}

\author{
Ting Wang $\mathbb{D}^{1,2}$ Kazuyuki Suzuki ${ }^{1},{ }^{1}$ Toshimi Chiba, ${ }^{2}$ Keisuke Kakisaka, \\ and Yasuhiro Takikawa ${ }^{1}$ \\ ${ }^{1}$ Division of Hepatology, Department of Internal Medicine, Iwate Medical University, Morioka, Iwate, Japan \\ ${ }^{2}$ Division of Internal Medicine, Department of Oral Medicine, Iwate Medical University, Morioka, Iwate, Japan
}

Correspondence should be addressed to Ting Wang; tingwang@iwate-med.ac.jp and Kazuyuki Suzuki; kasuzuki48@hotmail.co.jp

Received 1 June 2021; Revised 6 August 2021; Accepted 18 August 2021; Published 10 September 2021

Academic Editor: Amal Santra

Copyright (c) 2021 Ting Wang et al. This is an open access article distributed under the Creative Commons Attribution License, which permits unrestricted use, distribution, and reproduction in any medium, provided the original work is properly cited.

\begin{abstract}
Introduction. Ammonia is a key component in the pathogenesis of hepatic encephalopathy. Branched-chain amino acids (BCAA) have been reported to improve the symptoms of HE induced by hyperammonemia; however, we recently reported that ammonia increases intracellular levels of BCAA and exerts toxic effects on astrocytes. Objectives. This follow-up study was designed to confirm the direct effects of BCAA on human astrocytes and clarify their underlying mechanisms using metabolome analysis and evaluation of associated signaling. Methods. We performed cytotoxicity and cell proliferation tests on astrocytes following BCAA treatment with and without ammonium chloride $\left(\mathrm{NH}_{4} \mathrm{Cl}\right)$ and then compared the results with the effects of BCAA on hepatocytes and neurons. Subsequently, we used metabolomic analysis to investigate intracellular metabolite levels in astrocytes with and without BCAA treatment. Results. The astrocytes showed increased leakage of intracellular lactate dehydrogenase and reduced proliferation rate upon BCAA treatment. Interestingly, our analysis showed a BCAA-induced impairment of intracellular glycolysis/glyconeogenesis as well as amino acid and butyric acid metabolism. Furthermore, BCAA treatment was found to cause decreased levels of Glut-1 and phosphorylated GSK-3 $\beta$ and mTOR in astrocytes. Conclusions. Although further investigations of the effect of BCAA on human astrocytes with hyperammonemia are needed, our work demonstrates that BCAA supplementation has direct negative effects on astrocyte survival and intracellular metabolism.
\end{abstract}

\section{Introduction}

Plasma and brain amino acid (AA) imbalance, particularly low concentration of branched-chain amino acids (BCAA) and high concentration of aromatic amino acids (AAA) in plasma, closely participates in the pathogenesis of hepatic encephalopathy (HE) in liver cirrhosis (LC) and/or portalsystemic shunt, resulting in the disturbance of neurotransmission in the brain [1]. In general, orally administered BCAA is a clinically used nutritional therapy to alleviate protein-energy malnutrition (PEM) in patients with LC [2, 3]. Intravenous BCAA-enriched amino acid solution (Fischer's solution) is usually used in the initial stage of overt
HE, based on the false neurotransmitter theory [4-6]. Conversely, high AAA concentration in plasma induces neurological disturbances through the increased synthesis and release of false neurotransmitters in the brain, derived from AAA. BCAA supplementation can improve neurological disturbances by competitively reducing AAA concentrations in the brain via the blood-brain barrier (BBB).

Astrocytes play important roles in central nervous system (CNS) function and in maintaining the BBB through their "end feet," which interact with the structural components of the barrier. Recent studies have found that astrocytes determine both the functional and structural architecture of the adult brain [7]. Astrocyte structural 


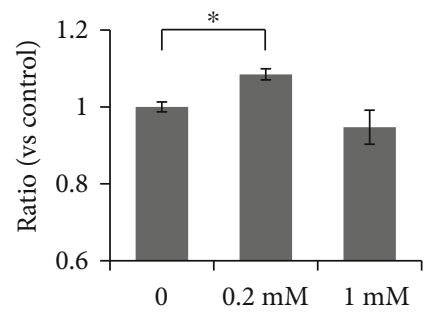

(a)

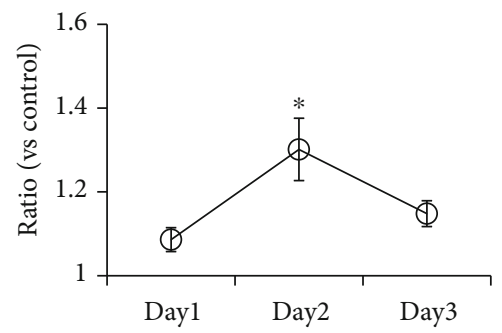

(c)

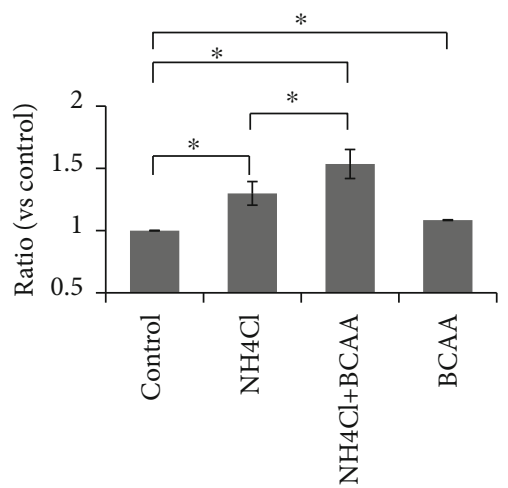

(e)

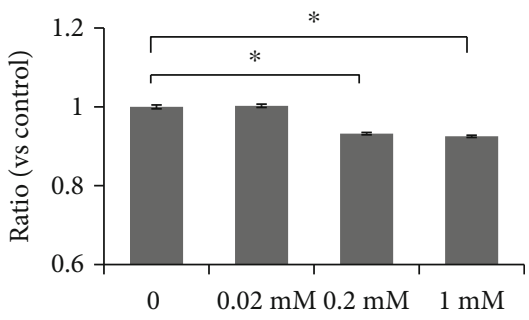

(b)

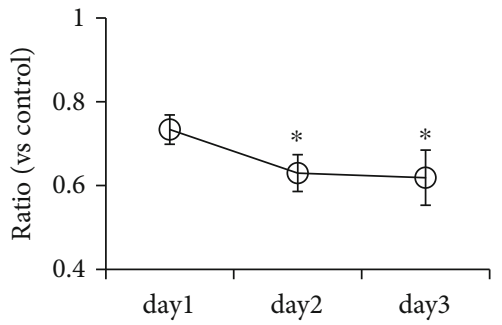

(d)

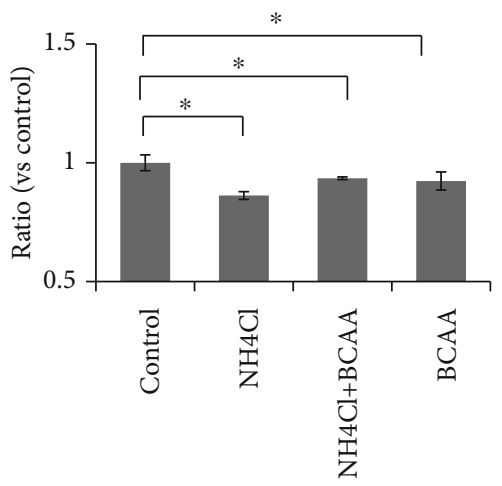

(f)

FIGURE 1: BCAA induce cytotoxicity and reduce astrocyte viability. LDH cytotoxicity assay (a) and evaluation of viable cells (b) among astrocytes following treatment with $0-1 \mathrm{mM}$ BCAA. HT22 cells (c) and HepG2 cells (d) were treated with 0.2 mM BCAA for 24, 48, and $72 \mathrm{~h}$ prior to calculating cell viability. LDH cytotoxicity assay (e) and evaluation of viable cells (f) among astrocytes, following treatment with $0.2 \mathrm{mM} \mathrm{BCAA}, 10 \mathrm{mM} \mathrm{NH}_{4} \mathrm{Cl}$, and BCAA+NH $\mathrm{NH}_{4} \mathrm{Cl}$ cotreatments. Control: no treatment with either BCAA or other reagents. Data are presented as the mean $\pm \mathrm{SD} ; n=3 ;{ }^{*} p<0.05$.

abnormalities and dysfunction may lead to disruption of the BBB [8]. Beyond that, astrocyte failure is implicated in the pathogenesis of neurological disorders such as astrocyte swelling in Alzheimer type 2 cells that are reported during the initiation of HE [9].

Ammonia is a key substance in the pathogenesis of HE $[10,11]$. We recently reported that ammonia increases the intracellular levels of BCAA and of their neurotoxic derivatives, such as 3-methyl-2-oxovaleric acid in human astrocytes. Our data suggest that BCAA can be toxicants and facilitate the cytotoxic activity of ammonia on astrocytes [12].

Previous research has highlighted the importance of evaluating the effects of BCAA overdose on the brain to determine BCAA threshold values for adverse effects [13]. However, no toxic BCAA accumulation has been detected in the brain except for that in the Maple Urine Symptom Disease (MUSD), a very rare genetic defect, which could not serve as a suitable model for BCAA overdose in healthy individuals [14]. Additionally, we previously determined the BCAA concentration in the peripheral blood, cerebrospinal fluid (CSF), and brain tissues of dogs with hyperammonemia as well as Eck fistula (a model of portal-systemic shunt); we found that the BCAA concentration in the blood was significantly lower than that in the control, while the BCAA concentration in CSF did not decrease significantly [15]. These findings have also been reported by other laboratories [16, 17]. Furthermore, BCAA concentration in brain tissue showed similar levels to the control [15]. These results suggest that BCAA levels may not decrease in the CSF and brain tissue of HE patients and that additional BCAA supplementation may increase the risk of overdose in such cases. Therefore, it is necessary to evaluate the effects of BCAA on the CNS with respect to HE treatment.

In this study, we aimed to shed light on the direct effects of BCAA on astrocyte survival and to identify the mechanism underlying the function of BCAA. 


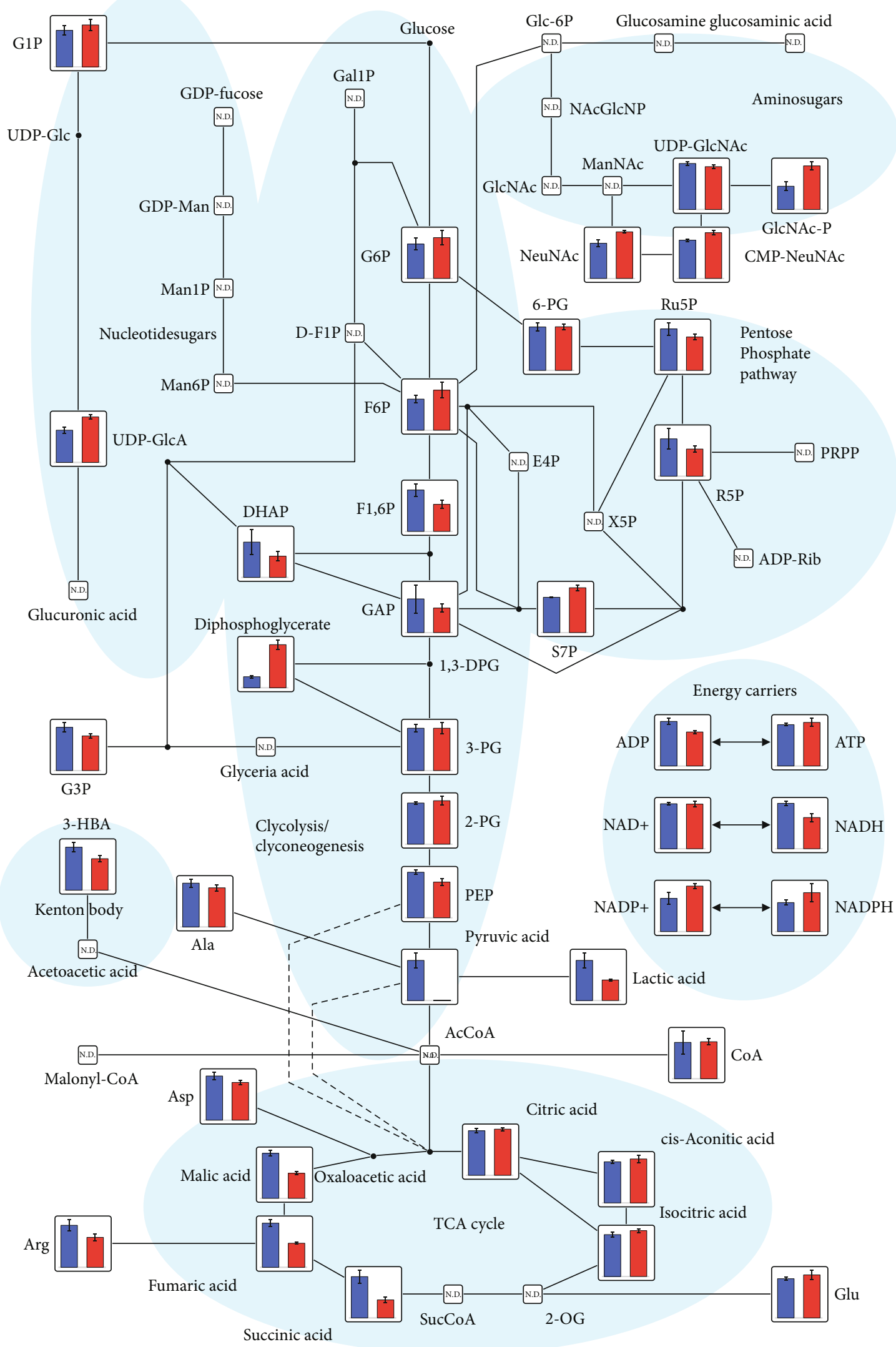

Figure 2: Map of central carbon metabolism pathways. Metabolites involved in the glycolysis/glyconeogenesis, TCA cycle, and central carbon metabolism are highlighted in the map. Blue bar: control (no treatment); Red bar: $\mathrm{NH}_{4} \mathrm{Cl}$ treatment. Data are presented as the mean \pm SD. $n=3$. 


\section{Methods}

2.1. Cell Culture and Treatments. The human astrocyte cell line Cryo NHA (CLCC-2565) was purchased from Lonza Walkersville, Inc. (Walkersville, MD, USA). Cells were grown in a medium based on the AGM Bullet Kit (CC3186) from the abovementioned company. The human hepatoblastoma cell line HepG2 was grown in DMEM supplemented with $10 \%$ fetal bovine serum (FBS, Thermo Fisher Scientific, New York, NY, USA). The mouse neuronal cell line HT22 was kindly provided by Dr. Takumi Satoh (Department of Welfare Engineering, Faculty of Engineering, Iwate University, Japan). Cells were grown in BCAAfree medium prior to BCAA treatment. Ammonium chloride $\left(\mathrm{NH}_{4} \mathrm{Cl}\right)$ was purchased from Sigma (St. Louis, $\mathrm{MO}$ ), and BCAA (leucine-isoleucine-valine, $2: 1: 1.2$ ) was kindly supplied by Ajinomoto Pharma Co. Ltd., Tokyo, Japan.

2.2. Evaluation of Viable Cells. Astrocytes, HepG2, and HT22 $\left(1 \times 10^{4}\right.$ cells/sample $)$ were treated with BCAA. Astrocytes were also treated with $\mathrm{NH}_{4} \mathrm{Cl}, \mathrm{BCAA}$, and a mixture of $\mathrm{NH}_{4} \mathrm{Cl}$ and BCAA. After $18 \mathrm{~h}$ of treatment, the number of viable astrocytes was evaluated using Cell Count Reagent SF (Nacalai Tesque Inc., Kyoto, Japan), as previously described [18]. $10 \mu \mathrm{L}$ of Cell Count Reagent SF was added to each well containing cultured astrocytes in $100 \mu \mathrm{L}$ medium. Cells were incubated for $1 \mathrm{~h}$, and then, absorbance was measured at $450 \mathrm{~nm}$ using a microplate photometer (IMMUNO-MINI NJ-2300, Inter Med Co., Tokyo, Japan). Changes in cell number were arbitrarily represented using the optical density ratio, defined as the number of stimulated cells divided by that of control cells.

2.3. Lactate Dehydrogenase (LDH) Cytotoxicity Assay. Astrocytes $\left(5 \times 10^{4}\right.$ cells/sample) were treated with $\mathrm{NH}_{4} \mathrm{Cl}$, BCAA, and a mixture of $\mathrm{NH}_{4} \mathrm{Cl}$ and BCAA. After $4 \mathrm{~h}$ of treatment, LDH activity was detected using an LDH Cytotoxicity Assay Kit (Cayman Chemical, Ann Arbor, MI, USA). Absorbance was read at $490 \mathrm{~nm}$ using a microplate reader (IMMUNOMINI NJ-2300, Inter Med Co., Tokyo, Japan).

2.4. Metabolome Analysis. Metabolomic screening was carried out through a facility service at Human Metabolome Technology (HMT) Inc., Tsuruoka, Japan. The experiment was carried out as described previously [12]. Astrocytes were treated with or without $0.2 \mathrm{mM}$ BCAA. After $4 \mathrm{~h}$ of treatment, a dish of cultured cells $\left(10^{6}\right.$ cells/sample) was used for the extraction of intracellular metabolites. The culture medium was aspirated from the dish, and cells were washed twice with a $5 \%$ mannitol solution $(10 \mathrm{~mL}$ first wash and $2 \mathrm{~mL}$ second wash). The cells were treated with $800 \mu \mathrm{L}$ of methanol and left at rest for $30 \mathrm{~s}$ to inactivate enzymes and extract the metabolites, according to the manufacturer's instructions. Next, the cell extracts were treated with $550 \mu \mathrm{L}$ of Milli-Q water containing internal standards (H3304-1002, Human Metabolome Technologies) and left at rest for another $30 \mathrm{~s}$. The extract was centrifuged at $2,300 \times g$ for $5 \mathrm{~m}$ at $4^{\circ} \mathrm{C}$, and $800 \mu \mathrm{L}$ of the upper aqueous layer was centrifugally filtered through a Millipore $5 \mathrm{kD}$ cutoff filter at $9,000 \times g$ for $120 \mathrm{~m}$ at $4^{\circ} \mathrm{C}$ to remove proteins.
TABLE 1: Relative changes of intracellular amino acids in astrocytes.

\begin{tabular}{lc}
\hline Name of amino acids & BCAA vs. control ratio \\
\hline Lysine & 0.7 \\
Arginine & $0.7^{*}$ \\
Histidine & 0.8 \\
Aspartate & 0.9 \\
Glutamate & 1.1 \\
Serine & 0.9 \\
Threonine & 0.9 \\
Asparagine & 0.9 \\
Glutamine & 0.9 \\
Alanine & 0.9 \\
Glycine & 0.9 \\
Valine & 0.8 \\
Isoleucine & 0.8 \\
Leucine & 0.8 \\
Phenylalanine & 0.7 \\
Tyrosine & $0.7^{*}$ \\
Tryptophan & $0.8^{*}$ \\
Methionine & 0.8 \\
Cysteine & 1.4 \\
Proline & 0.8 \\
\hline Data expresse &
\end{tabular}

Data expressed as the mean value $(n=3) .{ }^{*} p<0.05$ (Welch's $t$-test).

TABLE 2: Levels of metabolites related to butyric acid metabolism.

\begin{tabular}{lccc}
\hline Compound name & \multicolumn{3}{c}{$\begin{array}{c}\text { Comparative analysis } \\
\text { BCAA vs. control } \\
\text { pvalue }\end{array}$} \\
\hline 2-Hydroxyglutaric acid & 1.0 & 0.884 \\
3-Hydroxybutyric acid & 0.7 & 0.031 & $*$ \\
Fumaric acid & 0.5 & 0.008 & $*$ \\
GABA & 0.9 & 0.158 & \\
Glu & 1.1 & 0.298 & $*$ \\
Malic acid & 0.6 & 0.001 & $*$ \\
Pyruvic acid & $<1$ & N.A. \\
Succinic acid & 0.4 & 0.014 & $*$ \\
\hline
\end{tabular}

Data expressed as the mean value $(n=3) .{ }^{*} p<0.05$ and ${ }^{* *} p<0.01$ (Welch's $t$-test).

The filtrate was concentrated through centrifugation and resuspended in $50 \mu \mathrm{L}$ of Milli-Q water for the Capillary Electrophoresis Time-of-Flight Mass Spectrometry (CETOFMS) (Agilent Technologies, Waldbronn, Germany) analysis. The detailed methods for CE-TOFMS have been described in previous publications $[19,20]$.

Peaks were extracted using the automatic integration software MasterHands (Keio University, Tsuruoka, Japan) to obtain peak information, including $\mathrm{m} / \mathrm{z}$, migration time for CE-TOFMS measurements (MT), and peak area. Signal peaks corresponding to adduct ions and other products of known metabolites were excluded, and the remaining peaks were annotated as putative metabolites and their isotopic 


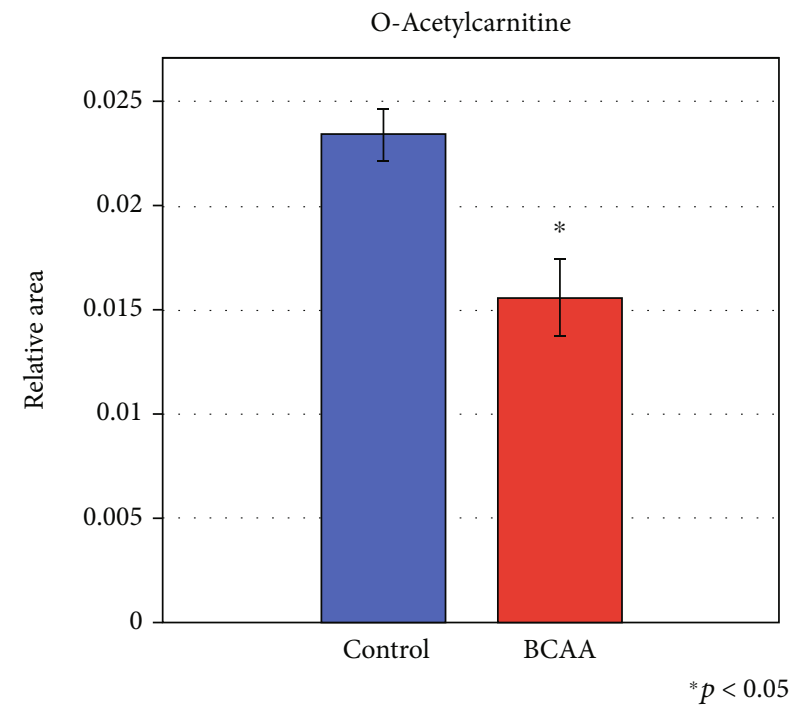

(a)

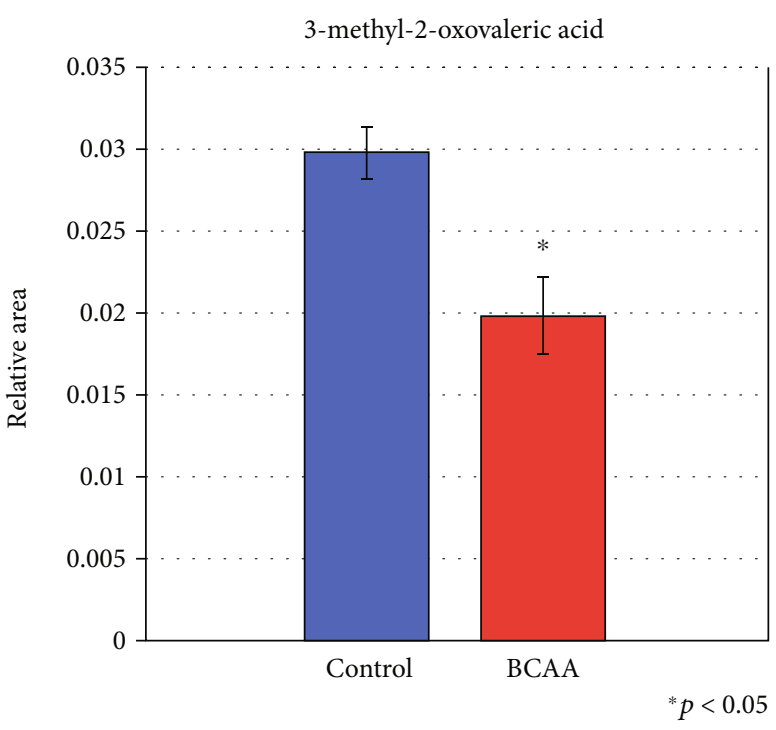

(b)

Figure 3: Effects of BCAA on the intracellular levels of neuroprotective and neural toxic metabolites. Intracellular levels of ALC (a) and 3methyl-2-oxovaleric acid (b) were measured and compared with those in the control (no treatment) group. Data are presented as the mean \pm SD. $n=3$.

ions from the HMT metabolic database based on their MT and $m / z$ values determined by TOFMS. The tolerance range for the peak annotation was configured at $\pm 0.5 \mathrm{~m}$ for MT and $\pm 30 \mathrm{ppm}$ for $\mathrm{m} / z$. In addition, peak areas were normalized against those of the internal standards, and the resultant relative area values were further normalized to sample amounts.

2.5. Western Blot Analysis. Standard Western blotting was carried out as described previously [21]. Total protein was isolated from hepatocytes, using a total protein extraction kit (BioChain Institute, Newark, CA, USA) according to the manufacturer's instructions. Ten micrograms of protein from each sample was separated using $10 \%$ sodium dodecyl sulfate polyacrylamide gel electrophoresis (SDS-PAGE) and electrotransferred to a polyvinylidene difluoride membrane. Immunoblotting was performed using specific antibodies against Glut-1 (Abcam, Cambridge, UK), p-GSK-3 $\beta$, and pmTOR (Cell Signaling Technology Japan, K.K., Tokyo, Japan). The antibody against $\beta$-actin was purchased from Santa Cruz Biotechnology (Santa Cruz, CA, USA). Immunoreactive bands were visualized with an enhanced chemiluminescence reagent (GE Healthcare, Little Chalfont, Buckinghamshire, UK) and quantified using ImageJ software.

2.6. Statistical Analysis. Three technical replicates were set for each experiment $(n=3)$. All data are expressed as the mean \pm standard deviation. Group comparisons were performed using Welch's $t$-test. Significance was defined as $p$ $<0.05$.

\section{Results}

3.1. BCAA Treatment Induces Cytotoxicity and Reduces Astrocyte Viability. Treatment of astrocytes with $0.2 \mathrm{mM}$
BCAA induced a mean increase of $14 \%(p<0.05)$ in LDH leakage (Figure 1(a)) and mean decrease of $9 \%(p<0.05)$ in viability compared with that of nontreated (control) cells (Figure 1(b)). Concerning other cell types, $0.2 \mathrm{mM}$ BCAA increased the proliferation of neuronal cells on the second day of treatment by approximately 30\% $\quad(p<0.05$, Figure $1(\mathrm{c})$ ), whereas it inhibited the proliferation of HepG2 cells $(35 \%$ and $38 \%$ mean reductions were observed on days 2 and 3, respectively; $p<0.05$; Figure 1(d)). Furthermore, BCAA and $\mathrm{NH}_{4} \mathrm{Cl}$ cotreatment resulted in enhanced cytotoxicity in astrocytes compared with both the control and $\mathrm{NH}_{4} \mathrm{Cl}$ treatment alone $(p<0.05$, Figure 1(e)). Although BCAA slightly improved cell viability, which was reduced by ammonia, cell viability was still significantly lower than that in the control cells $(p<0.05$, Figure $1(f))$.

\subsection{BCAA Treatment Affects Multiple Metabolic Pathways in} Astrocytes. To identify the mechanism underlying the toxic effects of BCAA in astrocytes, we investigated metabolite expression levels in both control cells and in BCAA-treated astrocytes. We observed a significant disturbance in multiple metabolic pathways. First, we detected alterations in metabolites linked to central carbon metabolism. Despite the significant increase $(p<0.05)$ in the 2,3-diphosphoglyceric acid level, metabolites including fructose-1,6-diphosphate, phosphoenolpyruvic acid, and acetic acid, which are critical in glycolysis/glycogenesis mechanisms, were significantly decreased $(p<0.05)$ in BCAA-treated cells, compared to the control. As for energy carriers participating in ATP generation, which is the major function of mitochondria, despite ATP and $\mathrm{NAD}^{+}$, which showed no obvious difference between the BCAA-treated and nontreated cells, all other components including ADP, fumaric acid, $\mathrm{NADH}$, and succinic acid were significantly decreased $(p<0.05)$ upon BCAA treatment. In addition, metabolites of the 

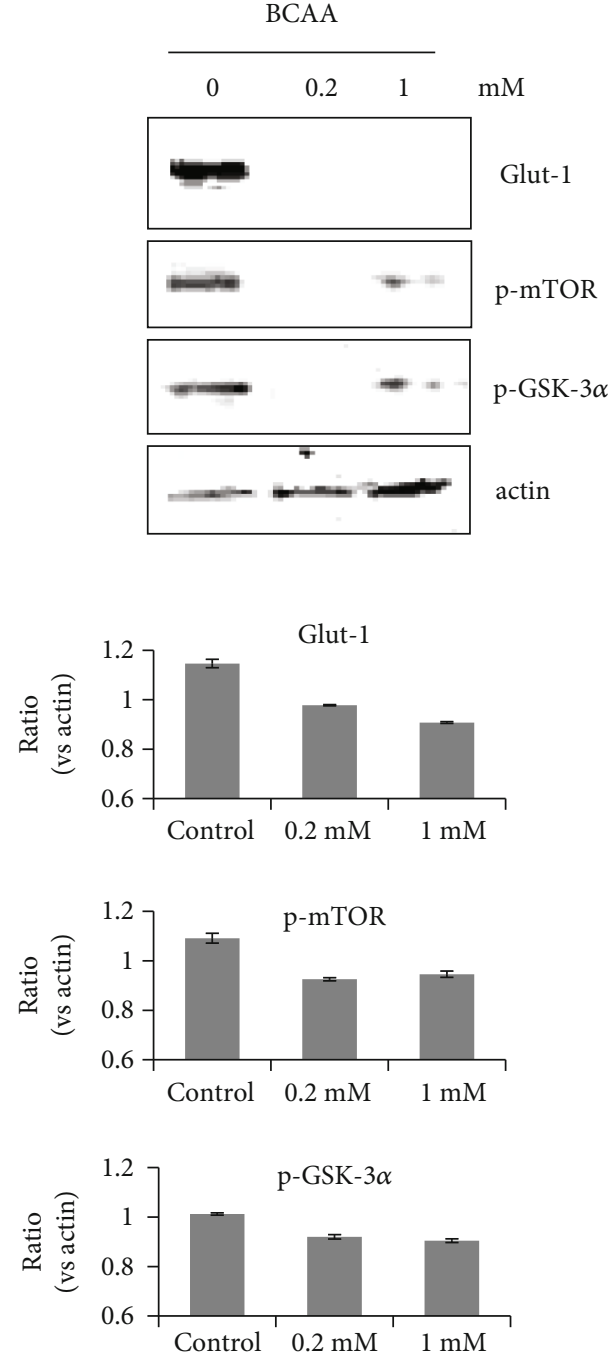

Figure 4: Effects of BCAA on the protein levels of Glut-1 and activation of GSK-3 $\beta$ and mTOR. Western blot analysis of Glut-1 $(60 \mathrm{kDa})$, phosphorylated GSK-3 $\beta \quad(46 \mathrm{kDa})$, and mTOR $(289 \mathrm{kDa})$ from extracted astrocytes, following $24 \mathrm{~h}$ treatment with $0.2 \mathrm{mM}$ BCAA. $\beta$-Actin $(43 \mathrm{kDa})$ was used as a loading control. The blots were cropped after stripping (Re-blot plus mild solution, Thermo Fisher Scientific Inc., Goteborg, Sweden) and restaining the same membrane. The protein bands from Western blot films were quantified with Image (NIH, USA). Control: no treatment. $n=3$.

tricarboxylic acid (TCA) cycle, such as malic acid, fumaric acid, and succinic acid, showed a significant decrease in BCAA-treated cells (Figure 2). Second, regarding intracellular protein metabolism, the relative levels of almost all intracellular amino acids, including BCAA, were decreased in BCAA-treated cells compared with the control (Table 1). Third, in terms of intracellular lipid metabolism, although levels of metabolites related to fatty acid synthesis, such as decanoic acid and lauric acid, were not significantly changed after BCAA treatment (data not shown), metabolites of the butyric (short-chain fatty acids) pathway, such as GABA, showed lower levels in BCAA-treated cells than in the control (Table 2). Additionally, the level of carnitine-derivative acetylcarnitine (ALC), which exerts neuroprotective effects by inducing ureagenesis and improving energy metabolism $[22,23]$, was decreased in BCAA-treated cells. Notably, 3methyl-2-oxovaleric acid, which is mainly produced from isoleucine and plays a critical role in neurological damage [24], was also decreased upon BCAA treatment (Figure 3).

\subsection{Effects of BCAA Treatment on GSK-3/mTOR Signaling in} Glut-1 Regulation. Glucose uptake, uniquely regulated by Glut-1, is an important function of astrocytes [25]. As our metabolomic analysis showed a disturbance in glycolysis/glyconeogenesis, we further investigated the possible effects of BCAA on Glut-1 production. The results of this experiment showed that an $18 \mathrm{~h}$ treatment with BCAA decreased Glut-1 protein levels. Moreover, signaling molecules linked to Glut-1 production and regulation, such as GSK-3 $\alpha / \beta$ and mTOR [26], were hypophosphorylated compared to the control (Figure 4).

\section{Discussion}

BCAA supplementation, especially BCAA infusion therapy, has been reported to improve the symptoms of HE induced by hyperammonemia [4-6]. However, the mechanisms underlying the direct effect of BCAA on the CNS have rarely been investigated. The vast majority of studies on ammonia toxicity in HE studies have focused on the effects of ammonia toxicity on astrocytes $[27,28]$. We have previously shown that ammonia increases cytotoxicity in human astrocytes and inhibits their proliferation [12]. In this study, we had initially predicted that BCAA would exert a protective effect on the astrocytes especially against hyperammonemia; however, our data strongly suggests that BCAA induces cytotoxicity, impairs astrocyte survival, and enhances the cytotoxic effect of $\mathrm{NH}_{4} \mathrm{Cl}$ on astrocytes. BCAA treatment slightly, but did not significantly, improved the antiproliferative effects of $\mathrm{NH}_{4} \mathrm{Cl}$ in astrocytes, as shown in Figure 1(f); although we could not identify the reason for this, it was clear that the effects of BCAA were too weak to aid total recovery of the level of proliferation of the astrocytes to that of the control.

On the other hand, BCAA has been reported to prevent hepatocarcinogenesis and prolong the survival of patients with cirrhosis [29]. Similarly, our data showed that BCAA inhibited the proliferation of human hepatoblastoma cells. Our in vitro data using two cell lines indicate that the anticancer effects of BCAA may be accompanied by side effects of neural damage. In addition, the negative impact of high BCAA levels on neurons is supported by recent findings based on cultured neurons and motor cortex slices from mice fed a BCAA-enriched diet [30]. Conversely, the present study showed a positive effect on the proliferation of mouse neuronal cells. Therefore, further studies are needed to confirm and clarify the effects of BCAA on both mouse and human neurons.

Metabolome analysis using blood and tissue samples has recently been developed, allowing to comprehensively evaluate the pathways of carbohydrate, AA, nucleotide, and lipid metabolism $[12,31]$. However, no reports have described the use of this method to measure the effects of BCAA on 
intracellular metabolites in astrocytes. Our previous study revealed that ammonia induces an abnormal intracellular increase in BCAA and its neural toxic derivatives, such as 3-methyl-2-oxovaleric acid [12]. Nonetheless, both BCAA and 3-methyl-2-oxovaleric acid were herein found to decrease with BCAA treatment. Our data therefore indicate that BCAA negatively affects astrocyte survival potentially by perturbing the cell metabolism, but not by toxic compound accumulation in astrocytes. In the present study, we elucidated the effects of BCAA on intracellular metabolism in this study with a single dose, single time point, and the limited repeated samples $(n=3)$ because of the high cost of the metabolism assay. Further studies should be conducted to completely understand the effects of BCAA on intracellular metabolism.

Astrocytes play a central role in controlling brain energy metabolism; this is supported by in vivo observations indicating that astrocytes are primarily responsible for glucose uptake in the brain [32] and that astrocytes take up half of the glucose from capillaries. Astrocytes are distinguished from neurons in the CNS based on their glucose uptake from the circulation through the Glut-1 glucose transporter. It has also been reported that Glut-1 silencing inhibits cell proliferation and glucose uptake [33]. In this study, Glut-1 protein levels in astrocytes decreased upon BCAA treatment. This indicates that the glucose uptake in astrocytes was impaired, which may be related to the disturbance of glycolysis/glycogenesis. The mTOR/GSK-3 pathway reportedly downregulates Glut-1 synthesis. However, upon BCAA treatment, the total protein levels of Glut-1 and the phosphorylation levels of mTOR and GSK-3 were all decreased. Our data suggests that the mTOR/GSK-3 pathway may not function upstream of Glut-1 in astrocytes upon BCAA treatment. Therefore, the mechanism of how BCAA affects glucose uptake and the correlation with the mTOR/GSK-3 signaling pathway should be further investigated.

In addition, we found evidence of decreased ALC levels upon BCAA treatment, which suggests a disturbance in intracellular carnitine metabolism. Carnitine deficiency has been proven to result in HE [34]; ALC plays an important role in fat metabolism and mitochondrial energy production [22] and has been considered a therapy for patients with overt $\mathrm{HE}$ or covert $\mathrm{HE}$ as it induces ureagenesis and improves energy metabolism [35]. We recently reported that carnitine reduced cytotoxicity and intracellular metabolism impairment caused by ammonia. We also found that carnitine treatment significantly increased the protein levels of Glut-1 in astrocytes (data not shown). Our data indicates the possibility that carnitine could prohibit the negative effects of BCAA on astrocytes.

In conclusion, although BCAA supplementation for $\mathrm{HE}$ treatment caused by LC and/or portal-systemic shunt has benefits that improve consciousness levels [36], the present study shows that BCAA has direct negative effects on astrocyte survival and intracellular metabolism, which suggests a limitation in HE. A further study to clarify the effects of BCAA on human astrocytes with hyperammonemia is needed, because BCAA supplementation showed no prevention for ammonia-induced toxicity in astrocytes.

\section{Data Availability}

The data used to support the findings of this study are included within the article.

\section{Conflicts of Interest}

All authors declare no conflict of interest.

\section{Acknowledgments}

This study was supported by Grants-in-Aid for Scientific Research (JP 25461009 and JP 19K07737) from the Japan Society for the Promotion of Science.

\section{References}

[1] J. E. Fischer and R. J. Baldessarini, "False neurotransmitters and hepatic failure," The Lancet, vol. 298, no. 7715, pp. 7580, 1971.

[2] Y. Muto, S. Sato, A. Watanabe et al., "Effects of oral branchedchain amino acid granules on event-free survival in patients with liver cirrhosis," Clinical gastroenterology and hepatology, vol. 3, no. 7, pp. 705-713, 2005.

[3] G. Marchesini, G. Bianchi, M. Merli et al., "Nutritional supplementation with branched-chain amino acids in advanced cirrhosis: a double-blind, randomized trial," Gastroenterology, vol. 124, no. 7, pp. 1792-1801, 2003.

[4] L. L. Gluud, G. Dam, M. Borre et al., "Oral branched-chain amino acids have a beneficial effect on manifestations of hepatic encephalopathy in a systematic review with metaanalyses of randomized controlled trials," The Journal of nutrition., vol. 143, no. 8, pp. 1263-1268, 2013.

[5] L. K. Bak, H. S. Waagepetersen, M. Sørensen et al., "Role of branched chain amino acids in cerebral ammonia homeostasis related to hepatic encephalopathy," Metabolic brain disease, vol. 28, no. 2, pp. 209-215, 2013.

[6] L. L. Gluud, G. Dam, I. Les et al., "Branched-chain amino acids for people with hepatic encephalopathy," The Cochrane database of systematic reviews., vol. 5, article CD001939, 2017.

[7] M. Nedergaard, B. Ransom, and S. A. Goldman, "New roles for astrocytes : Redefining the functional architecture of the brain," Trends in Neurosciences, vol. 26, no. 10, pp. 523-530, 2003.

[8] M. V. Sofroniew, "Astrocyte failure as a cause of CNS dysfunction," Molecular Psychiatry, vol. 5, no. 3, pp. 230-232, 2000.

[9] G. Seifert, K. Schilling, and C. Steinhauser, "Astrocyte dysfunction in neurological disorders: a molecular perspective," Nature Reviews. Neuroscience, vol. 7, no. 3, pp. 194-206, 2006.

[10] A. H. Lockwood, E. W. Yap, and W. H. Wong, "Cerebral ammonia metabolism in patients with severe liver disease and minimal hepatic encephalopathy," Journal of cerebral blood flow and metabolism, vol. 11, no. 2, pp. 337-341, 1991.

[11] R. F. Butterworth, "Pathogenesis of hepatic encephalopathy: new insights from neuroimaging and molecular studies," Journal of hepatology., vol. 39, no. 2, pp. 278-285, 2003.

[12] T. Wang, K. Suzuki, K. Kakisaka, M. Onodera, K. Sawara, and Y. Takikawa, "L-carnitine prevents ammonia-induced cytotoxicity and disturbances in intracellular amino acid levels in human astrocytes," Journal of gastroenterology and hepatology., vol. 34, no. 7, pp. 1249-1255, 2019. 
[13] J. D. Fernstrom, "Branched-chain amino acids and brain function," The Journal of nutrition., vol. 135, no. 6, pp. 1539S1546S, 2005.

[14] T. Kachaamy and J. S. Bajaj, "Diet and cognition in chronic liver disease," Current opinion in gastroenterology, vol. 27, no. 2, pp. 174-179, 2011.

[15] K. Suzuki, A. Kato, and M. Iwai, "Branched-chain amino acid treatment in patients with liver cirrhosis," Hepatology research, vol. 30S, pp. 25-29, 2004.

[16] A. Cascino, C. Cangiano, F. Fiaccadori et al., "Plasma and cerebrospinal fluid amino acid patterns in hepatic encephalopathy," Digestive diseases and sciences, vol. 27, no. 9, pp. 828$832,1982$.

[17] J. Butterworth, C. R. Gregory, and L. R. Aronson, "Selective alterations of cerebrospinal fluid amino acids in dogs with congenital portosystemic shunts," Metabolic brain disease, vol. 12, no. 4, pp. 299-306, 1997.

[18] M. Ishiyama, Y. Miyazono, K. Sasamoto, Y. Ohkura, and K. Ueno, "A highly water-soluble disulfonated tetrazolium salt as a chromogenic indicator for NADH as well as cell viability," Talanta, vol. 44, no. 7, pp. 1299-1305, 1997.

[19] T. Soga and D. N. Heiger, "Amino acid analysis by capillary electrophoresis electrospray ionization mass spectrometry," Analytical chemistry, vol. 72, no. 6, pp. 1236-1241, 2000.

[20] A. Hirayama, K. Kami, M. Sugimoto et al., "Quantitative metabolome profiling of colon and stomach cancer microenvironment by capillary electrophoresis time-of-flight mass spectrometry," Cancer research., vol. 69, no. 11, pp. 4918-4925, 2009.

[21] T. Wang, Y. Takikawa, K. Sawara, Y. Yoshida, and K. Suzuki, "Negative regulation of human astrocytes by interferon (IFN) $\alpha$ in relation to growth inhibition and impaired glucose utilization," Neurochemical Research, vol. 37, no. 9, pp. 1898-1905, 2012.

[22] F. M. Vaz and R. J. A. Wanders, "Carnitine biosynthesis in mammals," The Biochemical journal., vol. 361, no. 3, pp. 417-429, 2002.

[23] G. Therrien, C. Rose, J. Butterworth, and R. F. Butterworth, "Protective effect of L-carnitine in ammonia-precipitated encephalopathy in the portacaval shunted rat," Hepatology, vol. 25, no. 3, pp. 551-556, 1997.

[24] S. E. Snyderman, P. M. Norton, E. Roitman, and L. E. Holt Jr., "Maple syrup urine disease, with particular reference to dietotherapy," Pediatrics, vol. 34, pp. 454-472, 1964.

[25] A. Nehlig and J. A. Coles, "Cellular pathways of energy metabolism in the brain: is glucose used by neurons or astrocytes?," Glia, vol. 55, no. 12, pp. 1238-1250, 2007.

[26] C. L. Buller, R. D. Loberg, M. H. Fan et al., "A GSK3/TSC2/mTOR pathway regulates glucose uptake and GLUT1 glucose transporter expression," American journal of physiology Cell physiology, vol. 295, no. 3, pp. C836-C843, 2008.

[27] K. Ramarao, A. Jayakumar, and M. Norenberg, "Role of oxidative stress in the ammonia-induced mitochondrial permeability transition in cultured astrocytes," Neurochemistry International, vol. 47, no. 1-2, pp. 31-38, 2005.

[28] I. Zemtsova, B. Gorg, V. Keitel, H. J. Bidmon, K. Schror, and D. Haussinger, "Microglia activation in hepatic encephalopathy in rats and humans," Hepatology, vol. 54, no. 1, pp. 204$215,2011$.

[29] T. Kawaguchi, K. Shiraishi, T. Ito et al., "Branched-chain amino acids prevent hepatocarcinogenesis and prolong sur- vival of patients with cirrhosis," Clinical gastroenterology and hepatology, vol. 12, no. 6, pp. 1012-1018.e1, 2014, e1.

[30] R. de Simone, F. Vissicchio, C. Mingarelli et al., "Branchedchain amino acids influence the immune properties of microglial cells and their responsiveness to pro-inflammatory signals," Biochimica et Biophysica Acta, vol. 1832, no. 5, pp. 650-659, 2013.

[31] for "Precision Medicine and Pharmacometabolomics Task Group"-Metabolomics Society Initiative, R. D. Beger, W. Dunn et al., "Metabolomics enables precision medicine: "A White Paper, Community Perspective"," Metabolomics, vol. 12, no. 9, 2016.

[32] J. Chuquet, P. Quilichini, E. A. Nimchinsky, and G. Buzsaki, "Predominant enhancement of glucose uptake in astrocytes versus neurons during activation of the somatosensory cortex," Journal of Neuroscience, vol. 30, no. 45, pp. 1529815303, 2010.

[33] L. Al-Khalili, G. D. Cartee, and A. Krook, "RNA interferencemediated reduction in GLUT1 inhibits serum-induced glucose transport in primary human skeletal muscle cells," Biochemical and biophysical research communications, vol. 307, no. 1, pp. 127-132, 2003.

[34] B. N. Limketkai and S. D. Zucker, "Hyperammonemic encephalopathy caused by carnitine deficiency," Journal of general internal medicine, vol. 23, no. 2, pp. 210-213, 2008.

[35] M. Malaguarnera, "Acetyl-L-carnitine in hepatic encephalopathy," Metabolic brain disease, vol. 28, no. 2, pp. 193-199, 2013.

[36] H. Fukui, H. Saito, Y. Ueno et al., "Evidence-based clinical practice guidelines for liver cirrhosis 2015," Journal of gastroenterology, vol. 51, no. 7, pp. 629-650, 2016. 